\title{
INTRINSIC VOLUMES AND LINEAR CONTRACTIONS
}

\author{
GRIGORIS PAOURIS AND PETER PIVOVAROV
}

(Communicated by Thomas Schlumprecht)

\begin{abstract}
It is shown that intrinsic volumes of a convex body decrease under linear contractions.
\end{abstract}

Let $C \subset \mathbb{R}^{N}$ be a convex body and $B_{2}^{N}$ the Euclidean ball in $\mathbb{R}^{N}$. The Steiner formula expresses the volume of the Minkowski sum $C+\varepsilon B_{2}^{N}$ in terms of the intrinsic volumes $V_{0}, V_{1}, \ldots, V_{N}$ of $C$ :

$$
\operatorname{vol}_{N}\left(C+\varepsilon B_{2}^{N}\right)=\sum_{n=0}^{N} \omega_{n} V_{N-n}(C) \varepsilon^{n} .
$$

Here $\operatorname{vol}_{N}(\cdot)$ denotes $N$-dimensional Lebesgue measure and $\omega_{n}=\operatorname{vol}_{n}\left(B_{2}^{n}\right)$. Of particular interest are $V_{1}, V_{N-1}$ and $V_{N}$, which are multiples of the mean-width, surface area and volume, respectively. We refer the reader to 5 . for background on intrinsic volumes. In addition to their role in convex geometry, intrinsic volumes also appear in connection with Gaussian processes; see, e.g., 9], [10] and the references therein.

The purpose of this note is to prove the following.

Proposition 1.1. Let $C \subset \mathbb{R}^{N}$ be a convex body and let $S$ be a linear contraction; i.e., $\|S x\|_{2} \leqslant\|x\|_{2}$ for each $x \in \mathbb{R}^{N}$. Then for $n=1, \ldots, N$,

$$
V_{n}(S C) \leqslant V_{n}(C)
$$

The case of $V_{1}$ and arbitrary contractions (not necessarily linear) is well-studied [6. Theorem 2 in §5], [1, Theorem 1]; see also [2, p. 177]. Of course for $V_{N}$ one has $V_{N}(S C)=|\operatorname{det}(S)| \operatorname{vol}_{N}(C)$. For other intrinsic volumes, we were unable to find Proposition 1.1 in the literature but noticed that it follows from some results in [4] and thought it was worthwhile to show the details.

Received by the editors September 16, 2011.

2010 Mathematics Subject Classification. Primary 52A20, 52A39, 52A40.

The first-named author is supported by the A. Sloan Foundation, BSF grant 2010288, and the U.S. National Science Foundation, grant DMS-0906150.

The second-named author held a Postdoctoral Fellowship award from the Natural Sciences and Engineering Research Council of Canada and was supported by the Department of Mathematics at Texas A\&M University. 
Particularly useful for our purpose is the Gaussian representation of intrinsic volumes, as in [10]; see also [8. If $\Gamma_{N, n}=\left[\gamma_{i j}\right]$ is an $n \times N$ matrix with independent $\mathrm{N}(0,1)$ Gaussian entries, then the $n$-th intrinsic volume of $C \subset \mathbb{R}^{N}$ is given by

$$
V_{n}(C)=\frac{(2 \pi)^{n / 2}}{\omega_{n} n !} \mathbb{E} \operatorname{vol}_{n}\left(\Gamma_{N, n} C\right) .
$$

As in [4, we say that a function $F:\left(\mathbb{R}^{n}\right)^{N} \rightarrow \mathbb{R}^{+}$satisfies Groemer's Convexity Condition, or simply (GCC), if for every $z \in \mathbb{R}^{n}$ and for every $y_{1}, \ldots, y_{N} \in z^{\perp}$ the function $F_{Y}: \mathbb{R}^{N} \rightarrow \mathbb{R}^{+}$defined by

$$
F_{Y}(t)=F\left(y_{1}+t_{1} z, \ldots, y_{N}+t_{N} z\right)
$$

is even and convex. The latter definition was motivated by isoperimetric-type problems for random convex sets in [3]. In particular, by adapting [3, Lemma 3], it was shown in [4, Proposition 4.1] that for a convex body $C \subset \mathbb{R}^{N}$, the function $F:\left(\mathbb{R}^{n}\right)^{N} \rightarrow \mathbb{R}^{+}$defined by

$$
F\left(x_{1}, \ldots, x_{N}\right)=\operatorname{vol}_{n}\left(\left[x_{1} \ldots x_{N}\right] C\right),
$$

where $\left[x_{1} \ldots x_{N}\right]$ denotes the $n \times N$ matrix with columns $x_{1}, \ldots, x_{N}$, viewed as a linear operator from $\mathbb{R}^{N}$ to $\mathbb{R}^{n}$, satisfies (GCC). The latter property fits well with symmetrization techniques and can be used in various isoperimetric-type problems for the volume of random (and non-random) sets [4, Theorem 1.1].

For our present purpose, we require less than the (GCC) condition. In fact, we will use only the following consequence.

Lemma 1.2. If $F:\left(\mathbb{R}^{n}\right)^{N} \rightarrow \mathbb{R}^{+}$satisfies (GCC), then for any $x_{1}, \ldots, x_{N} \in \mathbb{R}^{n}$ and any $1 \leqslant j \leqslant N$, the function

$$
\mathbb{R} \ni s \mapsto F\left(x_{1}, \ldots, s x_{j}, \ldots, x_{N}\right)
$$

is convex.

The lemma is immediate since the restriction of a convex function to a line is itself convex.

Additionally, we will make use of the following elementary lemma (the proof is given in [4, Lemma 3.7]).

Lemma 1.3. Let $\rho: \mathbb{R}^{n} \rightarrow \mathbb{R}^{+}$be a function such that

$$
\mathbb{R} \ni s \mapsto \rho(s x)
$$

is convex for each $x \in \mathbb{R}^{n}$. If $X$ is a symmetric random vector with values in $\mathbb{R}^{n}$, then

$$
\mathbb{R}^{+} \ni s \mapsto \mathbb{E} \rho(s X)
$$

is an increasing function.

Here and elsewhere, we use the term "increasing" in the non-strict sense.

Proof of Proposition 1.1. As noted above, the function $F:\left(\mathbb{R}^{n}\right)^{N} \rightarrow \mathbb{R}^{+}$defined according to (1) satisfies (GCC). Let $g_{1}, \ldots, g_{N}$ denote the columns of the Gaussian random matrix $\Gamma_{N, n}$. If $g_{1}, \ldots, g_{N}$ are fixed, then

$$
\mathbb{R} \ni s \mapsto F\left(g_{1}, \ldots, g_{j-1}, s g_{j}, g_{j+1}, \ldots, g_{N}\right)
$$


is convex by Lemma 1.2 Letting $\mathbb{E}_{j}$ denote expectation with respect to $g_{j}$ and applying Lemma 1.3, we have that

$$
\mathbb{R}^{+} \ni s \mapsto \mathbb{E}_{j} F\left(g_{1}, \ldots, g_{j-1}, s g_{j}, g_{j+1}, \ldots, g_{N}\right)
$$

is an increasing function.

Suppose first that $S$ is represented by the $N \times N$ diagonal matrix $S=\operatorname{diag}(1, \ldots$, $\left.1, s_{j}, 1, \ldots, 1\right)$, where $s_{j} \in[0,1]$ is in the $j^{t h}$-column. Then

$$
\mathbb{E}_{j} F\left(g_{1}, \ldots, g_{j-1}, s_{j} g_{j}, g_{j+1}, \ldots, g_{N}\right) \leqslant \mathbb{E}_{j} F\left(g_{1}, \ldots, g_{j-1}, g_{j}, g_{j+1}, \ldots, g_{N}\right)
$$

and hence

$$
\begin{aligned}
(2 \pi)^{-n / 2} \omega_{n} n ! V_{n}(S C) & =\mathbb{E} \operatorname{vol}_{n}\left(\Gamma_{N, n} S C\right) \\
& =\mathbb{E} F\left(g_{1}, \ldots, g_{j-1}, s_{j} g_{j}, g_{j+1}, \ldots, g_{N}\right) \\
& \leqslant \mathbb{E} F\left(g_{1}, \ldots, g_{N}\right) \\
& =(2 \pi)^{-n / 2} \omega_{n} n ! V_{n}(C) .
\end{aligned}
$$

In the general case, using singular value decomposition, one writes $S=U D V^{T}$, where $D$ is the diagonal matrix $\operatorname{diag}\left(s_{1}, \ldots, s_{N}\right)$, and $U$ and $V$ are orthogonal. Since $S$ is a contraction, its singular values satisfy $0 \leqslant s_{i} \leqslant 1$ for $i=1, \ldots, N$. To conclude, we use the fact that intrinsic volumes are invariant under orthogonal transformations and apply the latter argument iteratively.

Remark 1.4. The latter proof uses ideas from [7, Lemma 2.7].

\section{ACKNOWLEDGEMENTS}

The authors thank A. Giannopoulos, R. Latała, R. Schneider, N. TomczakJaegermann and R. Vitale for helpful discussions.

\section{REFERENCES}

1. R. Alexander, Lipschitzian mappings and total mean curvature of polyhedral surfaces. I, Trans. Amer. Math. Soc. 288 (1985), no. 2, 661-678. MR776397 (86c:52004)

2. Yu. D. Burago and V. A. Zalgaller, Geometric inequalities, Grundlehren der Mathematischen Wissenschaften [Fundamental Principles of Mathematical Sciences], vol. 285, Springer-Verlag, Berlin, 1988. Translated from the Russian by A. B. Sosinskiı̌, Springer Series in Soviet Mathematics. MR 936419 (89b:52020)

3. H. Groemer, On the mean value of the volume of a random polytope in a convex set, Arch. Math. (Basel) 25 (1974), 86-90. MR0341286 (49:6036)

4. G. Paouris and P. Pivovarov, A probabilistic take on isoperimetric-type inequalities, Adv. Math. 230 (2012), 1402-1422. MR2921184

5. R. Schneider, Convex bodies: the Brunn-Minkowski theory, Encyclopedia of Mathematics and its Applications, vol. 44, Cambridge University Press, Cambridge, 1993. MR.1216521 (94d:52007)

6. V. N. Sudakov, Geometric problems in the theory of infinite-dimensional probability distributions, Proc. Steklov Inst. Math. (1979), no. 2, i-v, 1-178, Cover-to-cover translation of Trudy Mat. Inst. Steklov 141 (1976). MR.530375 (80e:60052)

7. S. J. Szarek, Spaces with large distance to $l_{\infty}^{n}$ and random matrices, Amer. J. Math. 112 (1990), no. 6, 899-942. MR1081810(91j:46023)

8. B. S. Tsirelson, A geometric approach to maximum likelihood estimation for an infinitedimensional Gaussian location. II, Theory Prob. Appl. 30, 820-828. Translation of Teor. Veroyatnost. i Primenen. 30 (1985), no. 4, 772-779. MR816291 (87i:62152)

9. R. A. Vitale, Intrinsic volumes and Gaussian processes, Adv. in Appl. Probab. 33 (2001), no. 2, 354-364. MR.1842297 (2002h:60076)

10. - On the Gaussian representation of intrinsic volumes, Statist. Probab. Lett. 78 (2008), no. 10, 1246-1249. MR2441470 (2009k:60090) 
Department of Mathematics, Texas A\&M University, College Station, Texas 778433368

E-mail address: grigoris@math.tamu.edu

Department of Mathematics, Texas A\&M University, College Station, Texas 778433368

E-mail address: ppivovarov@math.tamu.edu

Current address: Department of Mathematics, University of Missouri, Columbia, Missouri 65211

E-mail address: pivovarovp@missouri.edu 